\title{
Work on preeclampsia rewarded by the ASN
}

$T$ he American Society of Nephrology (ASN) recently honored S. Ananth Karumanchi (Figure 1)for his work on preeclampsia, a serious complication of approximately $5 \%$ of all pregnancies, with its Young Investigator Award.

Preeclampsia is characterized by a dramatic increase in blood pressure and excess protein in the urine during pregnancy. If left untreated, it can lead to seizures, coma, and occasionally death of the mother and/or fetus. The only cure is delivery of the fetus and placenta. Although this protects the mother, it can have severe consequences for the infant, who is delivered pre-term. The JCI had the opportunity to speak with Karumanchi about his work and plans for the future.

JCI: How did you feel when you learned you would receive the 2008 ASN Young Investigator Award?

Karumanchi: I entered biomedical research not for the awards but for the opportunity to work on unsolved medical mysteries. That said, awards are nice, since it is recognition from your peers that you are doing something right. This ASN award in particular was important to me, as this society took a chance on me early in my career, by giving me a small grant that funded my initial studies in preeclampsia when I was having difficulty obtaining NIH funding.

JCI: You are being recognized for your work investigating the triggers of preeclampsia. What do you consider your most crucial work in this area?

Karumanchi: It had been known for decades that the fundamental problem in preeclampsia is a defective placenta, and that the signs and symptoms of the disease markedly improve after delivery of the placenta. Over the last seven years, my laboratory has characterized two soluble proteins that likely mediate the signs and symptoms of preeclampsia. Our manuscript in the JCI in 2003 (1) was critical, but so were some of the clinical studies that followed.

JCI: When and how did you become interested in preeclampsia?

Karumanchi: Although I trained as a nephrologist, my postdoctoral work was in the area of renal cancer and angiogenesis. When I started my independent research career, I was interested in studying the molecular basis of kidney diseases thought to have a primary vascular basis. Preeclampsia caught

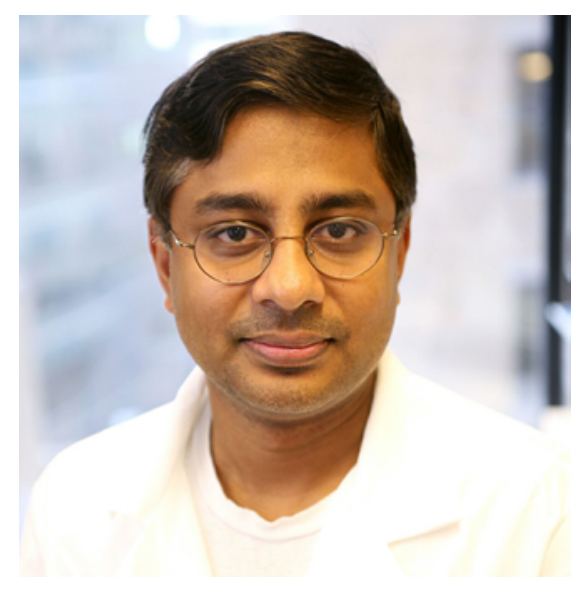

\section{Figure 1}

S. Ananth Karumanchi, recipient of the 2008 ASN Young Investigator Award. Image credit: Kaye Evans-Lutterodt/PR Newswire, copyright HHMI.

my attention, as its phenotypes - hypertension and proteinuria - are largely renal phenotypes and its etiology was known to be the placenta, an organ where physiological angiogenesis was first described in the 1930s.

JCI: In what direction is your research heading next?

Karumanchi: It turns out that soluble anti-angiogenic proteins are made in normal pregnancy and that in preeclampsia they are made prematurely and at higher levels. We want to understand what regulates the production of these proteins during normal pregnancy, in the hope that this knowledge gets us closer to what is happening in preeclampsia. We are also working in the laboratory on developing a safe therapeutic that blocks the actions of these toxic factors, which we hope may one day allow obstetricians to safely prolong pregnancy.

JCI: Do you think your work can be translated into the clinic?

Karumanchi: The research in my laboratory has provided a strong biologic rationale for developing a therapeutic and identified several potential targets. Unfortunately, because of the difficulties of performing a clinical trial in pregnant women, there has been little interest from pharmaceutical companies. Using rodent models we have developed two potential methods for treating this condition. However, we do not know if they will be safe for use in human pregnancies. I am hopeful that if we can demonstrate safety and efficacy in primates, we will have the have the opportunity to test these drugs in clinical trials. On the diagnostics side, several large diagnostic companies are currently performing clinical studies to assess whether the proteins we identified can be used to predict and diagnose preeclampsia.

JCI: You obtained your MD from the University of Madras, India. How easy was the transition from life in India to life in the US?

Karumanchi: As the language of instruction was English during medical school and the clinical training was superb, I did not have much trouble during my residency or clinical fellowship. My biggest shock was walking into a US research laboratory for the first time and discovering how underprepared I was to take on the challenge of medical research. Although my renal fellowship only required two years in the laboratory, I elected to spend an additional two years to ensure I had the necessary skills and technical courage to survive when I started my own laboratory. In retrospect, having obtained an NIH K08 award quite early in my fellowship made this decision to spend extra time in the laboratory easy.

JCI: Your research was highlighted in a 2006 article in The New Yorker (2). Do you think this raised the profile of your work or the profile of the disease?

Karumanchi: I was indeed fortunate that someone like Jerome Groopman, one of the best medical writers, highlighted our work. The article very nicely depicts the serendipity of medical research and how so many people with different backgrounds were involved in this effort. The article certainly made the public more aware of the disease, and I received several messages from patients and/or relatives encouraging me to continue what I was doing. It also convinced my father, who never really understood why I was not a "real" doctor seeing patients daily, that I was doing something important.

\section{Karen Honey}

1. Maynard, S.E., et al. 2003. Excess placental soluble fms-like tyrosine kinase 1 (sFlt1) may contribute to endothelial dysfunction, hypertension, and proteinuria in preeclampsia. J. Clin. Invest. 111:649-658.

2. Groopman, J. July 24, 2006. The preeclampsia puzzle. The New Yorker. http://www.newyorker. com/archive/2006/07/24/060724fa_fact. 